\title{
Electrochemical Properties of Silver Nanoparticle Doped Aminosilica Nanocomposite
}

\author{
Yong-Jae Choi and Tzy-Jiun M. Luo \\ Department of Materials Science and Engineering, North Carolina State University, Raleigh, NC 27695, USA \\ Correspondence should be addressed to Yong-Jae Choi, yongjae.choi@gmail.com and \\ Tzy-Jiun M. Luo, mark_luo@ncsu.edu
}

Received 2 May 2011; Revised 23 June 2011; Accepted 23 June 2011

Academic Editor: Shen-Ming Chen

Copyright ( $) 2011$ Y.-J. Choi and Tzy-J.M. Luo. This is an open access article distributed under the Creative Commons Attribution License, which permits unrestricted use, distribution, and reproduction in any medium, provided the original work is properly cited.

\begin{abstract}
The electrochemical properties of silver nanoparticle $(d \sim 5 \mathrm{~nm})$ synthesized within aminosilica film from spontaneous reduction reaction were examined using cyclic voltammetry and the results show that the nanocomposite film exhibits similar redox property as solution-synthesized silver nanoparticles when measuring in phosphate buffer solution and its redox potentials were found to be sensitive to the presence of chloride ions. It also shows that silver nanoparticles and hydrolyzed aminosilica increase the electron diffusivity of the aminosilica film. Both results confirm that an accurate reference electrode suitable for microfluidic devices can be created simply by treating an aminosilica-coated electrode with a silver nitrate solution. Furthermore, a humidity sensor based on silver-silica nanocomposite film has also been demonstrated.
\end{abstract}

\section{Introduction}

The sol-gel materials have been widely used as an immobilization matrix to encapsulate variety of chemical species for optical, chemical, biochemical, and energy applications [1$3]$. One limit of these materials is their low electrical conductivity due to the intrinsic property of silica itself. To overcome this drawback, there have been increasing interests to create nanocomposite materials based on sol-gel method that contains metallic components. These two components silica and metal have to work together to exhibit the desired properties that can never be achieved by single component alone.

Fabrication of metal-silica composite electrode has been reported using numerous methods [4-6]. However, the majority of the methods involve multiple steps due to the requirement of both reduction and immobilization of metallic nanoparticles $[7,8]$. We have reported a simple and one step approach to synthesize aminosilica materials with immobilized silver nanoparticles distributed at a nanometer scale (nanocomposite) [9]. Here, we examine the electrochemical properties of the nanocomposite film produced from spontaneous reduction of silver. The effects of silver nanoparticles on electron transfer within the nanocomposite film were also examined in phosphate buffer and ferricyanide solutions. Each experiment in different solution shows distinct redox characteristic, indicating that this nanocomposite prepared by simple dip-coat method can be used as a sensitive electrode.

\section{Experimental Procedure}

2.1. General Preparation of Silver Nanoparticle Doped Silica $\mathrm{Gel}$. The silver nanoparticle doped aminosilica film was created fallowing simple two steps approach (Figure 1). The fluorine doped tin oxide (FTO) glass, purchased from Hartford Glass Co., was used as the working electrode. This FTO electrode was cleaned with acetone and methanol in ultrasonic bath for 10 minutes and then dried under compressed air. The surface was further treated in $\mathrm{O}_{2}$-plasma cleaner for 3 minutes to improve the adhesion of silica film on the surface. The thin film of bis[3-(trimethoxysilyl)propyl] ethylenediamine(enTMOS) was created on FTO glass from a methanol solution containing $0.1 \mathrm{M}$ enTMOS by dip coating or spin coating. After the coating was dried in the air, the aminosilica-coated FTO glass was immerged into 


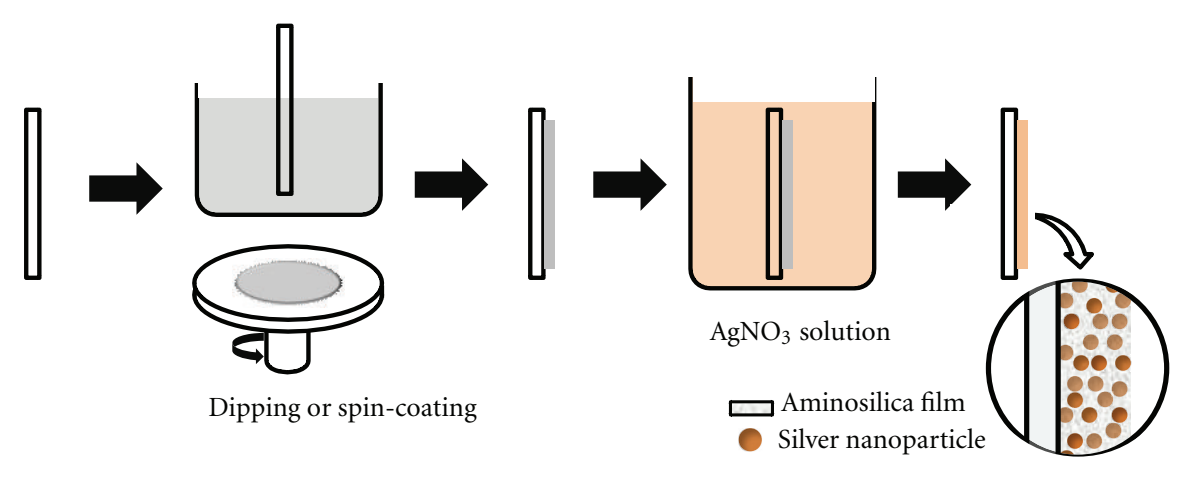

FIGURE 1: Schematic diagram of creating a silver-aminosilica nanocomposite electrode.

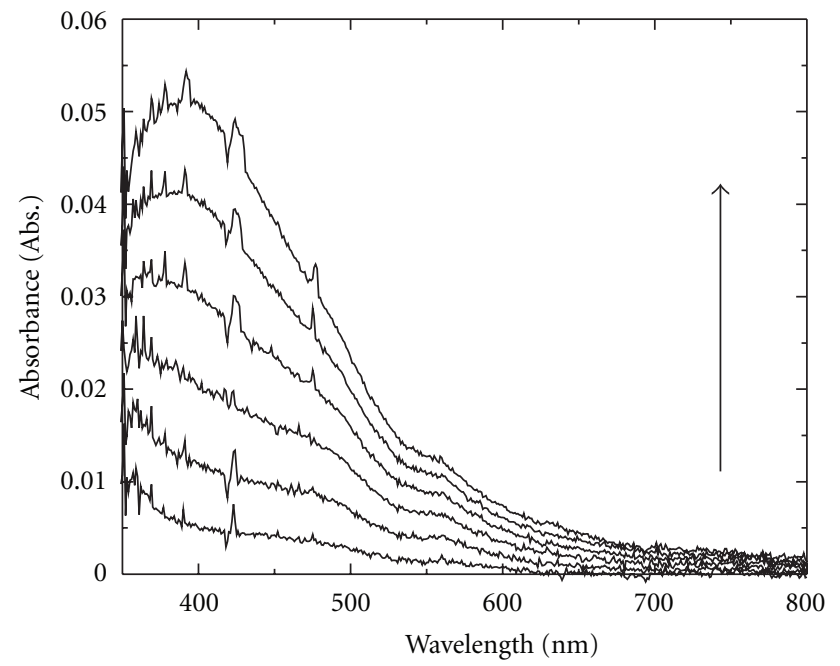

FIGURE 2: Time-lapse UV-VIS absorption spectra of silver nanoparticles synthesized in an aminosilica (enTMOS) film. Each spectrum was measured at each 10 minute interval for a total of one hour in a methanol solution containing $0.1 \mathrm{M}$ silver nitrate $\left(\mathrm{AgNO}_{3}\right)$. The film was created on top of a FTO electrode followed by immersion in an $\mathrm{AgNO}_{3}$ solution to create silver nanoparticles. The absorbance peak at $400 \mathrm{~nm}$ indicates the presence of nanoparticles.

a $0.1 \mathrm{M} \mathrm{AgNO}_{3}$ methanol solution for $60 \mathrm{~min}$. The resulting electrode exhibited light brownish-yellow color, which indicates the presence of silver nanoparticles inside of the enTMOS film. Then, electrode were washed with methanol several times to remove the excessive $\mathrm{AgNO}_{3}$ solution.

2.1.1. UV-VIS Spectroscopy. UV-VIS spectroscopy method was used to monitor the formation of silver nanoparticles within the aminosilica matrix. The baseline of the spectra was calibrated before the measurement. The FTO electrode was placed inside a cuvette so that it is perpendicular to the beam path. Then, the cuvette was later filled with $0.1 \mathrm{M}$ $\mathrm{AgNO}_{3}$ methanol solution and the measurement was taken at each 10 minutes for a total of an hour.

2.1.2. Transmission Electron Microscopy. TEM images were performed on a JEOL 2010 with an accelerating voltage of

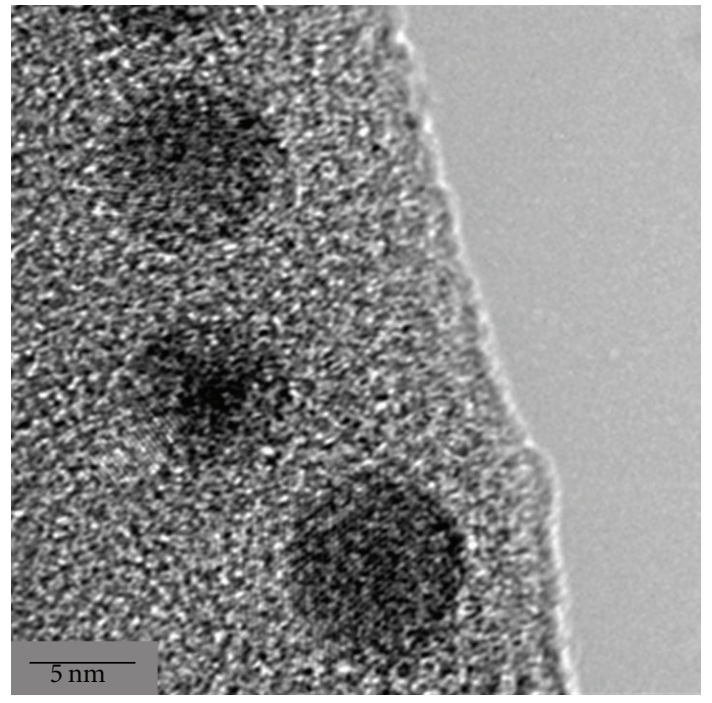

FIGURE 3: TEM cross-sectional image of an aminosilica film that contains silver nanoparticles. This image indicates that the synthesized nanoparticles within the film are $\sim 5 \mathrm{~nm}$ in diameter. The lattice space is $0.237 \mathrm{~nm}$, which corresponds to the $d$-space of the (111) face $(\sim 0.236 \mathrm{~nm})$.

$200 \mathrm{kV}$. A 200 mesh gold grid was used. First, an enTMOS film was created on TEM grid by dipping the grid in a diluted enTMOS solution and dried in the air, then this enTMOS coated grid was dipped in a $0.1 \mathrm{M} \mathrm{AgNO}_{3}$ solution for an hour. The grid was gently rinsed with methanol solutions and allowed the sample to dry.

2.1.3. Cyclic Voltammetry ( $C V$ ). The Faraday MP (Obbligato Objectives, Inc.) potentiostat was used to perform the electrochemical measurements. Unless it is otherwise stated, all measurements were performed at $50 \mathrm{mV} \mathrm{s}^{-1}$ scan rate. A three-electrode flow cell was constructed as previously described $[9,10]$ and the nanocomposite-coated FTO electrode was used as the working electrode. The reference electrode and counter electrode were silver chloride coated silver wire $(\mathrm{Ag} / \mathrm{AgCl})$ and platinum foil, respectively. The flow cell formed a sandwich device, and a round shape 


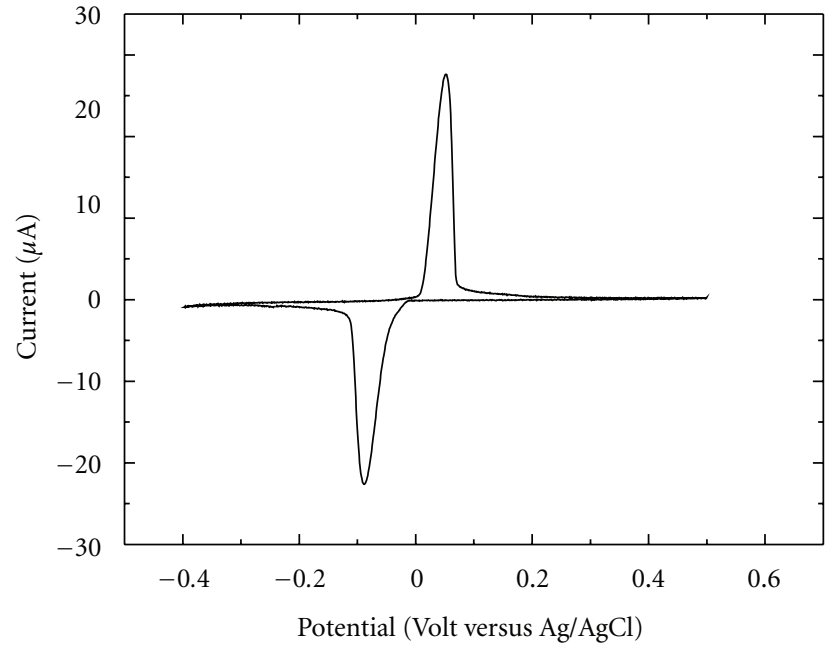

(a)

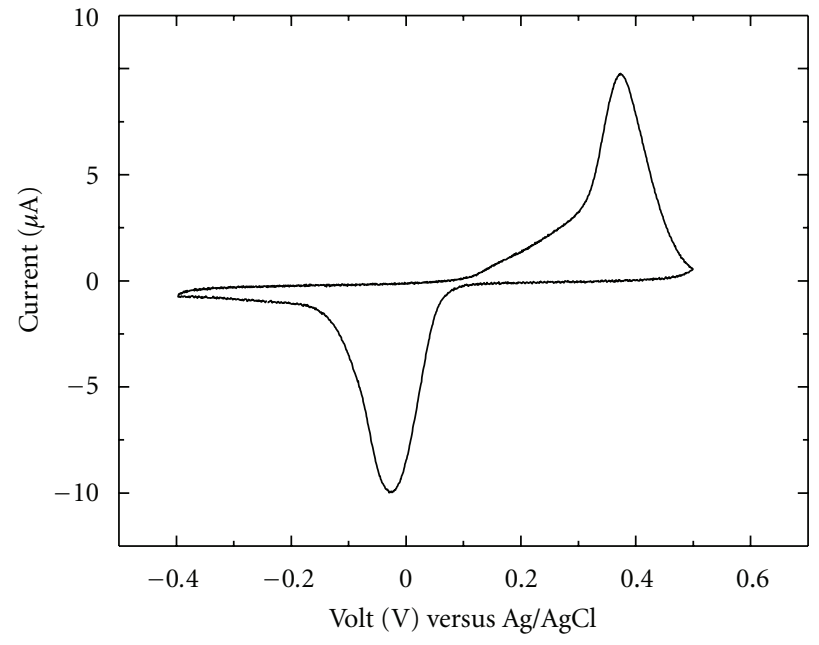

(b)

FIGURE 4: (a) The cyclic voltammogram (CV) of silver nanoparticle doped enTMOS nanocomposite film. The measurement was performed in a phosphate buffer saline solution containing $137 \mathrm{mM}$ chloride ions with a scan rate of $50 \mathrm{mV} \mathrm{s}^{-1}$. (b) The cyclic voltammogram of the same nanocomposite film in a phosphate buffer without chloride ions $\left(50 \mathrm{mV} \mathrm{s}^{-1}\right)$. The peaks appear broader, due to that silver oxide and silver phosphate are both involved in the redox reactions.

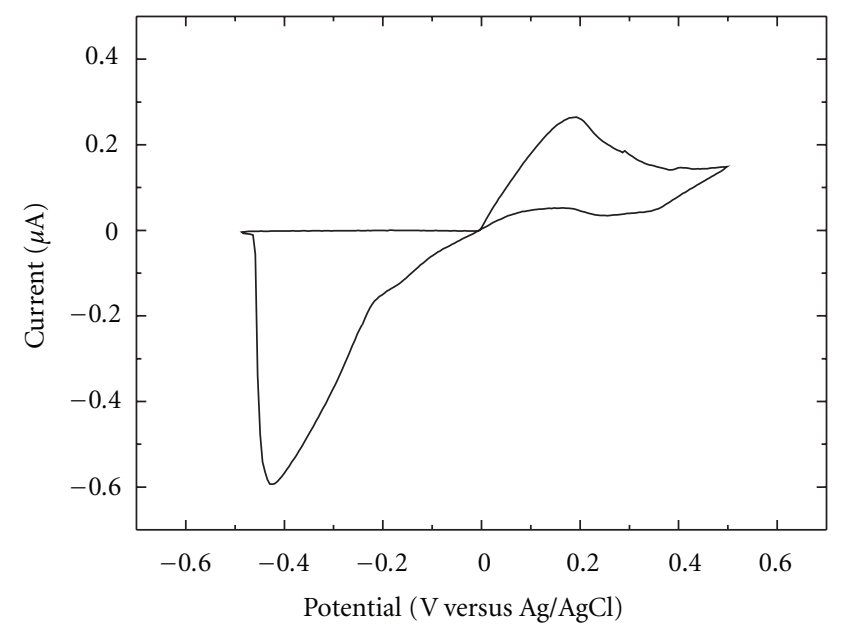

(a)

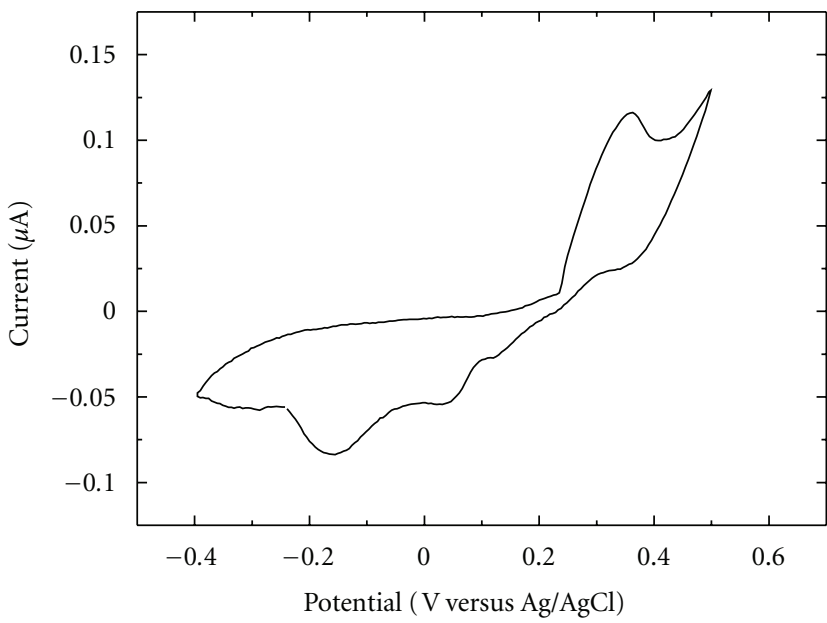

(b)

FIGURE 5: (a) The cyclic voltammogram of a silver wire electrode in a phosphate-buffered saline solution. The oxidation peak near zero volt corresponds to the formation of silver chloride on the surface of electrode. (b) CV of the bulk silver electrode performed in a phosphate buffer. The broadening of peaks indicates more complicated redox reactions occur on surface, which also involve silver oxide.

area on the working electrode about $2.68 \mathrm{~mm}^{2}$ was allowed to contact with the electrolyte. The flow cell holds about $0.1 \mathrm{~mL}$ of electrolyte. Here, electrolyte includes phosphate buffer (with/ without chloride ions) or sodium nitrate with ferricyanide ions depending on the required experimental setup.

The CV measurement with ferricyanide ions was done in the same electrochemical cell containing a solution of $10 \mathrm{mM}$ ferricyanide and $0.1 \mathrm{M}$ sodium nitrate as electrolyte. Aminosilica electrode either with or without silver nanoparticles was fabricated according to the method described in previous section. The enTMOS film with ferricyanide was prepared by dipping the film into a $10 \mathrm{mM}$ ferricyanide ion solution and washed with deionized water before the CV test.

\subsubsection{Fabrication of Silver-Aminosilica Nanocomposite Film} for Humidity Sensing. To create a nanocomposite film that is suitable for humidity testing, self-assembly process similar to the previous report was used here [11]. A polypropylene substrate was immersed in the solution containing $\mathrm{AgNO}_{3}$ and enTMOS premixed at a 1:4 molar ratio. After this composite film was created, the substrate was removed from the solution, washed, and then dried. Later, the film was peeled off from the substrate and attached to a glass substrate with both ends of the film connected by a conductive carbon 


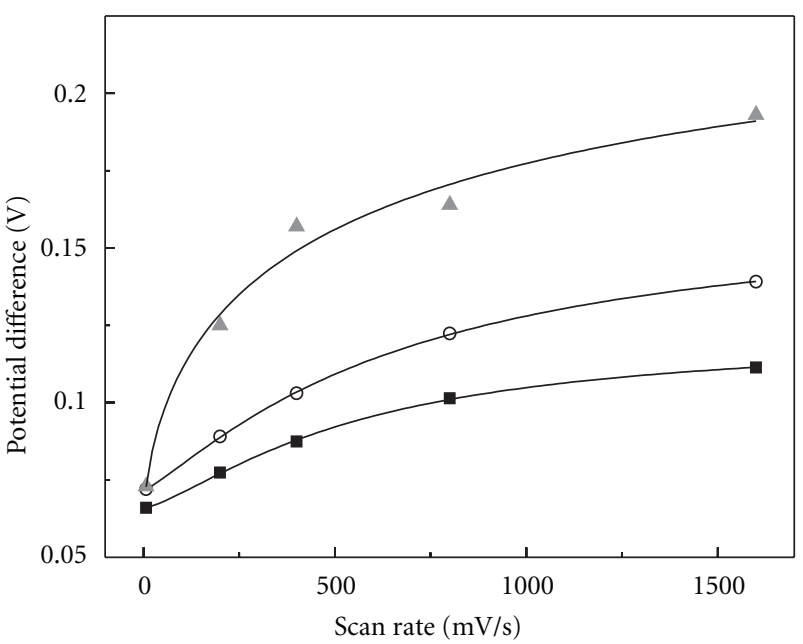

- AgNP-enTMOS film

- enTMOS film

$\triangle$ FTO

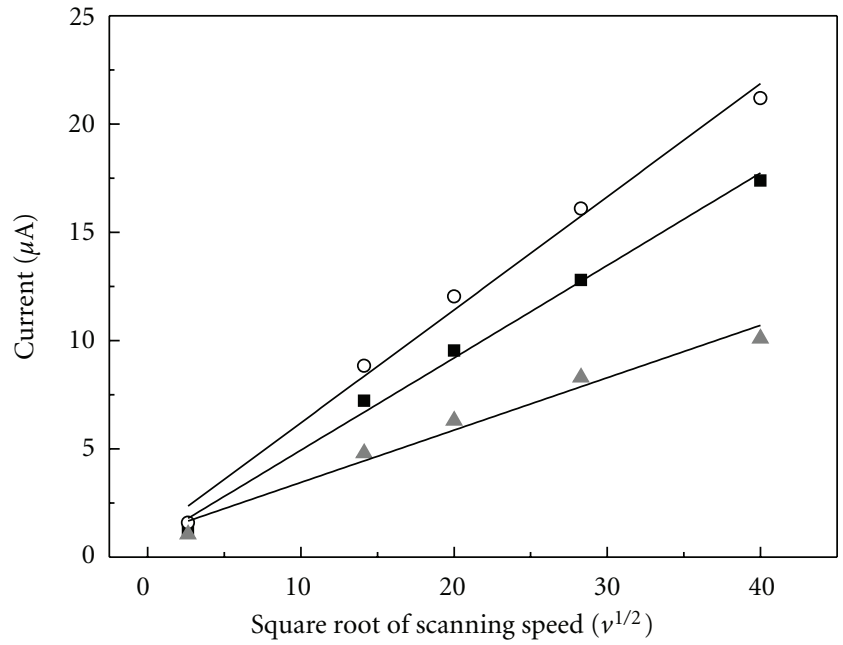

- AgNP-enTMOS film

- enTMOS film

$\triangle$ FTO

(a)

(b)

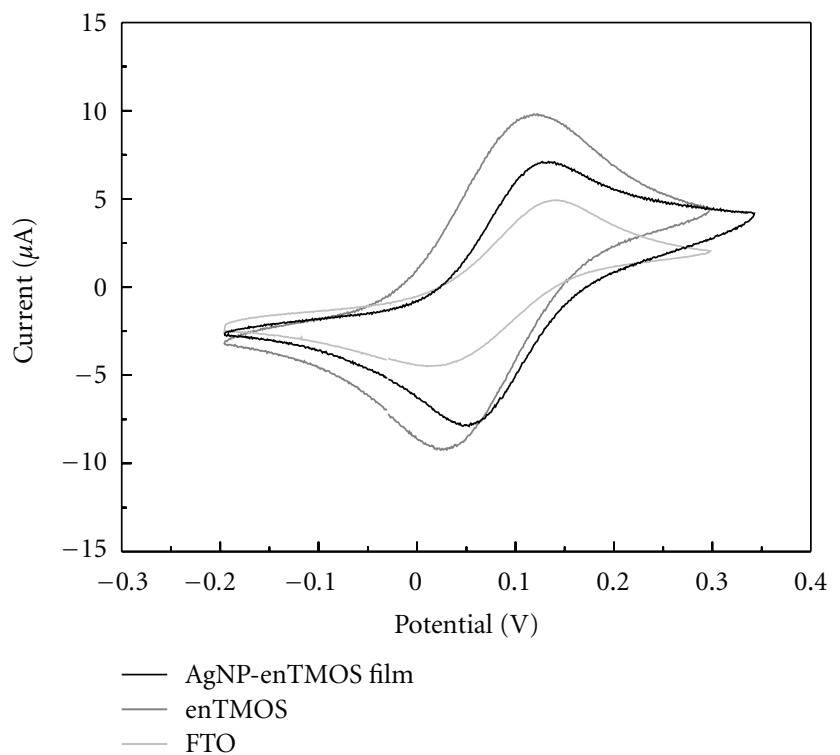

(c)

FIGURE 6: (a) Peak-peak separation between the oxidation and reduction potentials versus scan rate for enTMOS films with and without silver nanoparticles and a blank FTO electrode. CV was measured in a $10 \mathrm{mM}$ ferricyanide solution containing $0.1 \mathrm{M} \mathrm{NaNO}_{3}$ as electrolyte. (b) The peak current of oxidation/reduction potential of $10 \mathrm{mM}$ ferricyanide versus square root of scan rate at 7, 200, 400, 800, and 1600 $\mathrm{mV} \mathrm{s}^{-1}$. The results were compared to a blank FTO electrode. (c) The corresponding CV of the three electrodes under a scan rate of $200 \mathrm{mV} \mathrm{s}{ }^{-1}$ are shown.

tape. Humidity measurement was performed inside a glove box, where humidity was adjusted by mixing desired amount of air and dry nitrogen gas. Humidity inside the chamber was monitored using a portable humidity sensor (Model RH390, Extech Instruments). The electrical resistance of the film was recorded by a Keithley 614 electrometer.

\section{Results and Discussion}

Time-lapse UV-Vis spectroscopy in Figure 2 shows that silver nanoparticles were slowly produced inside the nanoporous aminosilica film when it was immersed in silver nitrate solution, causing the transparent film to turn into brownish yellow color. The increasing absorbance at $\sim 400 \mathrm{~nm}$ wavelength indicates that the concentration of silver nanoparticles has increased over time. Size of the nanoparticles within aminosilica was determined to be $\sim 5 \mathrm{~nm}$ by TEM (Figure 3), which is thought to be limited by the pore size of aminosilica matrix. These results indicate that the creation of nanoparticles inside the silica materials can be simply achieved by dipping enTMOS films into a silver ion solution. Although TEM image shows individual silver 


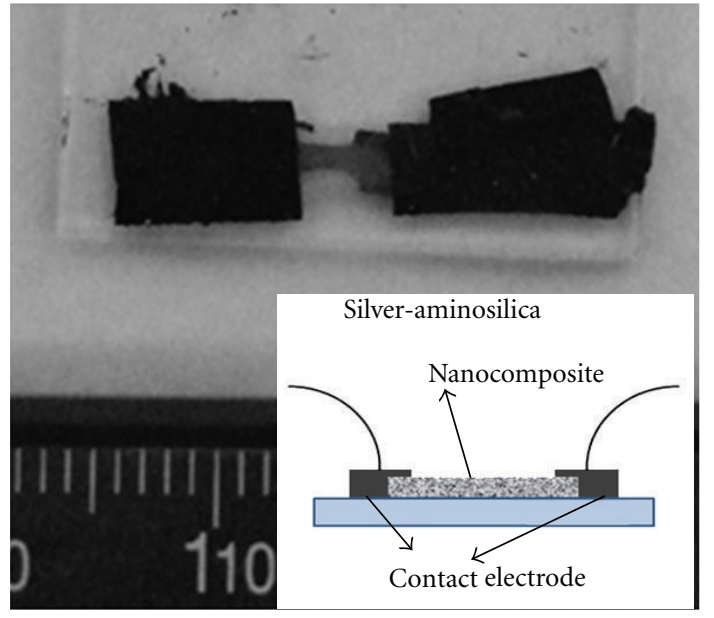

(a)

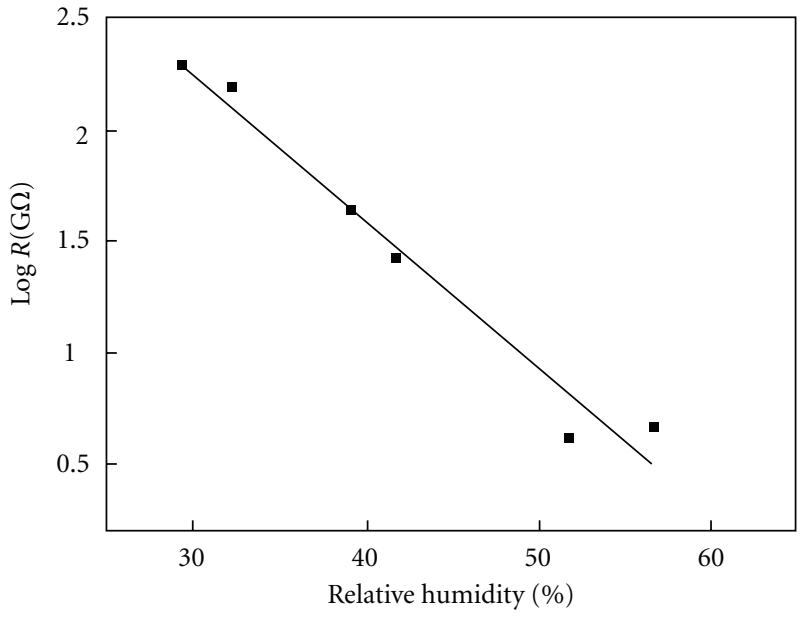

(b)

Figure 7: (a) A humidity sensor based on silver-aminosilica nanocomposite. The nanocomposite film was mounted on a glass substrate and secured by two carbon tapes at the both ends. The inset illustrates the layout of the device. (b) The logarithm of electrical resistance of the nanocomposite is inversely proportional to relative humidity of the air.

nanoparticles are separated, which indicates nanoparticles do not directly contact with the FTO conducting layer, cyclic voltammetry (CV) measurement of the film in phosphate-buffered saline $(1 \times \mathrm{PBS}, 137 \mathrm{mM} \mathrm{NaCl})$ shows that the nanocomposite film exhibit very sharp redox current $( \pm 25 \mu \mathrm{A})$ with a narrow voltage range at $\sim 0 \mathrm{~V}$ versus $\mathrm{Ag} / \mathrm{AgCl}$ reference electrode (Figure 4(a)). The oxidation and reduction potentials of silver nanoparticles are $0.05 \mathrm{~V}$ and $-0.09 \mathrm{~V}$, corresponding to $\mathrm{Ag}+\mathrm{Cl}^{-} \rightarrow \mathrm{AgCl}$ and $\mathrm{AgCl} \rightarrow \mathrm{Ag}$, respectively [12]. Such behavior is similar to the reported silver nanoparticles results $[13,14]$, indicating pore-size limited nanoparticles produced from spontaneous reduction reaction within the aminosilica matrix are suitable for electrochemical application. Due to the high surface area to volume ratio of the nanoparticles, the peak current is very high with a very narrow peak width. On the contrary, when immersed in phosphate solution (without $\mathrm{NaCl}$ ), redox reactions are associated with $\mathrm{Ag} \rightarrow \mathrm{Ag}_{2} \mathrm{O}$ and $\mathrm{Ag}_{2} \mathrm{O} \rightarrow \mathrm{Ag}$ at $0.37 \mathrm{~V}$ and $-0.03 \mathrm{~V}$ [15-17], respectively (Figure 4(b)). Two redox peaks are broader than that in Figure 4(a) with maximum current at $\pm 10 \mu \mathrm{A}$.

Compared to silver nanocomposite, a silver wire ( $d \sim 0.7 \mathrm{~mm}$ ) working electrode shows much broader redox peaks in both PBS and phosphate solutions. The redox peaks are not symmetrical and the peak-peak separation is always greater than the CV graph of silver nanoparticle film even at the same scanning rate $\left(50 \mathrm{mV} \mathrm{s}^{-1}\right)$, which indicates a slow and complex reaction $[12,18]$ (Figures 5(a) and 5(b)). The redox reactions on the surface of silver electrode were found to be more complicated than the nanocomposite samples. Because there was no redox chemical species from the solution directly involved, all redox reactions observed on CV come from silver itself.

The electron transfer rate between redox chemicals and silver nanoparticles were monitored using ferricyanide electrolyte. The aminosilica films containing silver nanoparticle were tested on the home-built electrochemical flow cell that contains ferricyanide solution. Because the aminosilica film is coated on the electrode surface, the CV curve does not follow the Nernst equation. The results show that silver nanoparticles within the aminosilica film improve the electron transfer rate, as indicated by its smallest difference of oxidation and reduction potential (Figure 6(a)). Since the kinetics of electron transfer between ferricyanide and FTO electrode is constant, the potential difference increases with the scan rate. Compared to blank FTO electrode, pure aminosilica film also shows an improved electron transfer due to the positively charged matrix that attracts ferricyanide ions and facilitates the electron transfer.

Both pure aminosilica and nanocomposite films show higher diffusion rate of ferricyanide at the electrode, as seen on the steep slope on the current and scan rate plot (Figure 6(b)). The current density of both aminosilica and nanocomposite films is also higher than that of a blank FTO electrode, which confirms that high concentration of ferricyanide ions is trapped inside the positively charged aminosilica matrix. However the overall redox current of nanocomposite film is lower than that of the pure aminosilica film due to that pores of aminosilica film are occupied by silver nanoparticles (Figure 6(c)), which may reduce the diffusion of ferricyanide ions. Although the diffusion rate is low within the aminosilica film, silver nanoparticles enhance the electron transfer between FTO electrode and redox chemicals by allowing the electrons to hop through.

The nanoporous structure of aminosilica could trap water molecule from the air and affects the surface conductivity under the presence of silver nanoparticles. This property was further utilized to demonstrate the concept of humidity sensing [19]. A thin enTMOS film that was created by mixing silver nitrate and aminosilane at a $1: 4$ mole ratio was tested in different humid environments. The results show that the electrical resistance of the nanocomposite decreases 
with humidity (Figure 7). To determine its relationship, logarithm of resistance values was plotted against relative humidity with a sensitivity ratio of $0.066 \log (\mathrm{G} \Omega) \mathrm{RH}^{-1}$.

\section{Conclusions}

The electrochemical properties of silver nanoparticles synthesized via spontaneous reduction of silver nitrate inside an aminosilica-coated electrode were examined by cyclic voltammetry and the results show same performance as those of solution-synthesized silver nanoparticles. Compared to regular silver electrode, nanocomposite electrode also exhibits higher redox currents density with a much narrower halfpeak potential width. This proves that silver nanoparticles directly synthesized from aminosilica matrix can serve as the reference electrode. In addition, the humidity-sensitive characteristics of the nanocomposite film has been further utilized to demonstrate its humidity sensing ability.

\section{References}

[1] V. B. Kandimalla, V. S. Tripathi, and H. X. Ju, "Immobilization of biomolecules in sol-gels: biological and analytical applications," Critical Reviews in Analytical Chemistry, vol. 36, no. 2, pp. 73-106, 2006.

[2] X. Ren, X. Meng, D. Chen, F. Tang, and J. Jiao, "Using silver nanoparticle to enhance current response of biosensor," Biosensors and Bioelectronics, vol. 21, no. 3, pp. 433-437, 2005.

[3] S. Rezaei-Zarchi, A. A. Saboury, P. Norouzi et al., "Use of silver nanoparticles as an electron transfer facilitator in electrochemical ligand-binding of haemoglobin," Journal of Applied Electrochemistry, vol. 37, no. 9, pp. 1021-1026, 2007.

[4] J. Wang and P. V. A. Pamidi, "Sol-gel-derived gold composite electrodes," Analytical Chemistry, vol. 69, no. 21, pp. 44904494, 1997.

[5] C. R. Raj and B. K. Jena, "Efficient electrocatalytic oxidation of NADH at gold nanoparticles self-assembled on three-dimensional sol-gel network," Chemical Communications, no. 15, pp. 2005-2007, 2005.

[6] D. H. Lin, Y. X. Jiang, Y. Wang, and S. G. Sun, "Silver nanoparticles confined in SBA-15 mesoporous silica and the application as a sensor for detecting hydrogen peroxide," Journal of Nanomaterials, vol. 2008, no. 1, Article ID 473791, 10 pages, 2008.

[7] B. Akkopru and C. Durucan, "Preparation and microstructure of sol-gel derived silver-doped silica," Journal of Sol-Gel Science and Technology, vol. 43, no. 2, pp. 227-236, 2007.

[8] L. Armelao, G. Bottaro, R. Campostrini et al., "Synthesis and structural evolution of mesoporous silica-silver nanocomposites," Nanotechnology, vol. 18, no. 15, Article ID 155606, 8 pages, 2007.

[9] Y. J. Choi, U. Huh, and T. J. M. Luo, "Spontaneous formation of silver nanoparticles in aminosilica," Journal of Sol-Gel Science and Technology, vol. 51, no. 1, pp. 124-132, 2009.

[10] T. J. M. Luo, Y. Choi, and U. Huh, "A microfluidic device for opto-electrochemical sensing," in Proceedings of the NSTI Nanotechnology Conference and Trade Show, pp. 363-366, May 2007.

[11] Y. J. Choi and T. J. M. Luo, "Self-assembly of silver-aminosilica nanocomposites through silver nanoparticle fusion on hydrophobic surfaces," ACS Applied Materials \& Interfaces, vol. 1, no. 12, pp. 2778-2784, 2009.
[12] Z. J. Jiang, C. Y. Liu, and Y. J. Li, "Electrochemical studies of silver nanoparticles tethered on silica sphere," Chemistry Letters, vol. 33, no. 5, pp. 498-499, 2004.

[13] G. F. Wang, W. Wang, J. F. Wu, H. Y. Liu, S. F. Jiao, and B. Fang, "Self-assembly of a silver nanoparticles modified electrode and its electrocatalysis on neutral red," Microchimica Acta, vol. 164, no. 1-2, pp. 149-155, 2009.

[14] M. Marcaccio, M. Margotti, M. Montalti, F. Paolucci, L. Prodi, and N. Zaccheroni, "Self-assembly of monolayer-coated silver nanoparticles on gold electrodes. An electrochemical investigation," Collection of Czechoslovak Chemical Communications, vol. 68, no. 8, pp. 1395-1406, 2003.

[15] L. Guo, J. Nie, B. Du, Z. Peng, B. Tesche, and K. Kleinermanns, "Thermoresponsive polymer-stabilized silver nanoparticles," Journal of Colloid and Interface Science, vol. 319, no. 1, pp. 175$181,2008$.

[16] G. F. Wang, M. G. Li, Y. C. Gao, and B. Fang, "Amperometric sensor used for determination of thiocyanate with a silver nanoparticles modified electrode," Sensors, vol. 4, no. 9, pp. 147-155, 2004.

[17] G. Chang, J. Zhang, M. Oyama, and K. Hirao, "Silver-nanoparticle-attached indium tin oxide surfaces fabricated by a seed-mediated growth approach," Journal of Physical Chemistry B, vol. 109, no. 3, pp. 1204-1209, 2005.

[18] G. S. Popkirov, M. Burmeister, and R. N. Schindler, "Electrode potential redistribution during silver oxidation and reduction in alkaline solution," Journal of Electroanalytical Chemistry, vol. 380, no. 1-2, pp. 249-254, 1995.

[19] B. N. Pal, T. K. Kundu, S. Banerjee, and D. Chakravorty, "Humidity sensing by nanocomposites of silver in silicate glass ceramics," Journal of Applied Physics, vol. 93, no. 7, pp. 42014206, 2003. 


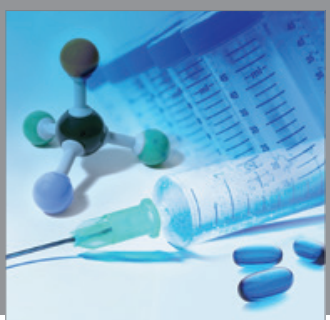

International Journal of

Medicinal Chemistry

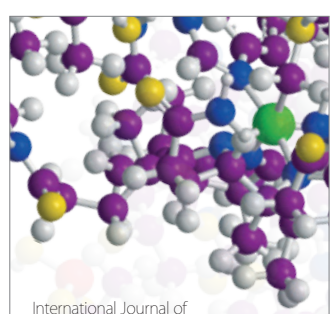

Carbohydrate Chemistry

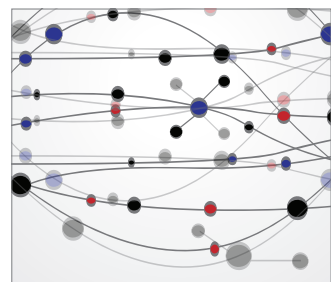

The Scientific World Journal
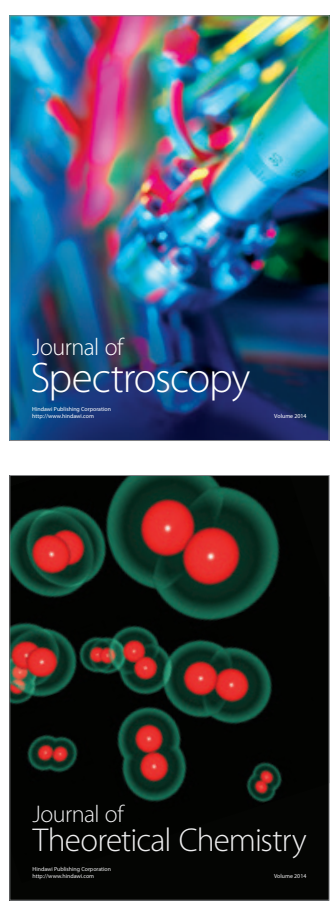
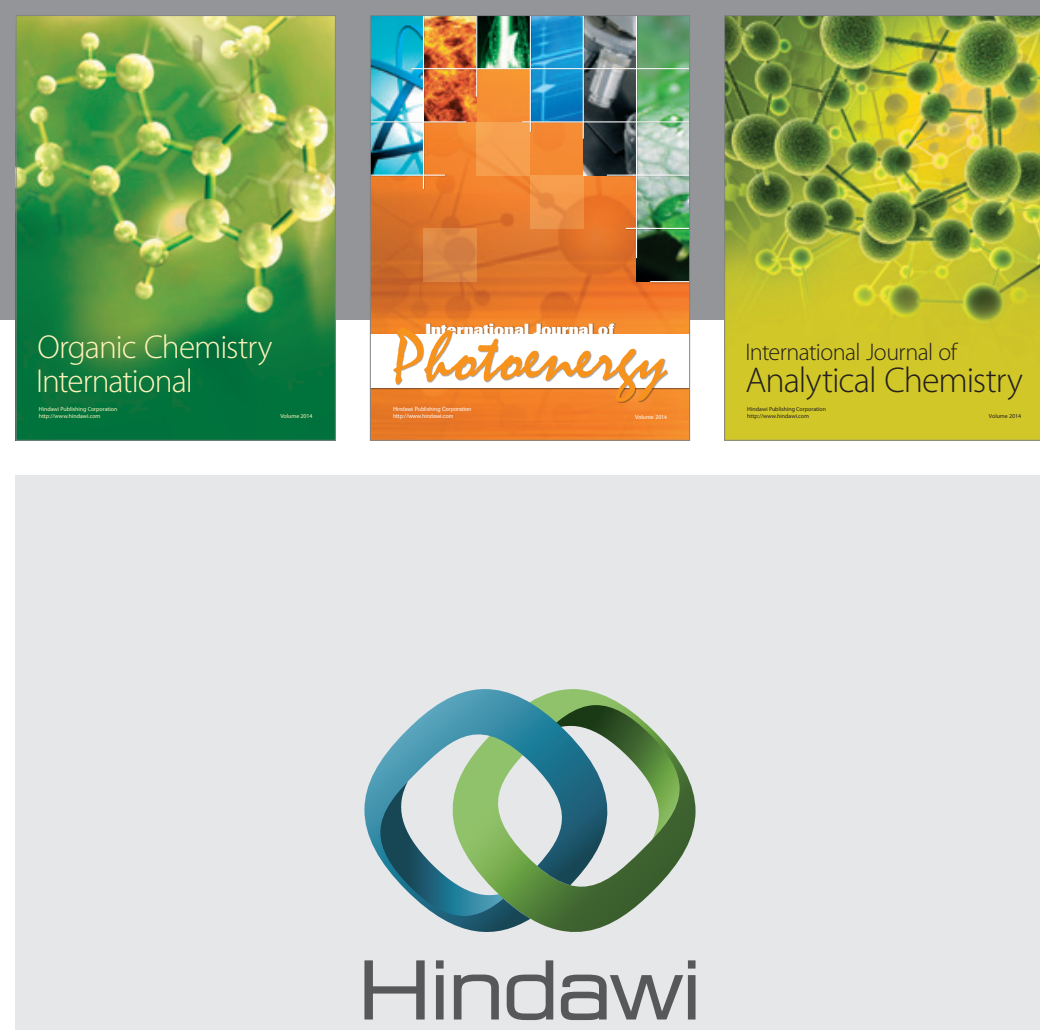

Submit your manuscripts at

http://www.hindawi.com
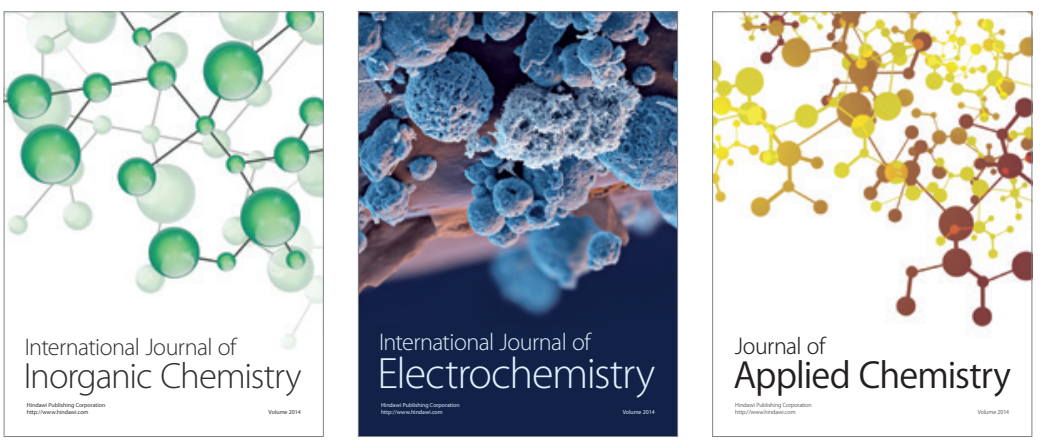

Journal of

Applied Chemistry
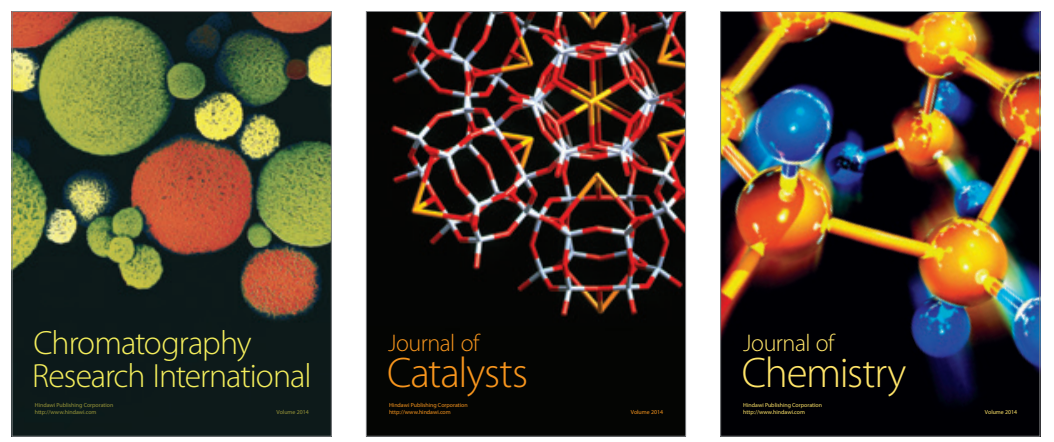
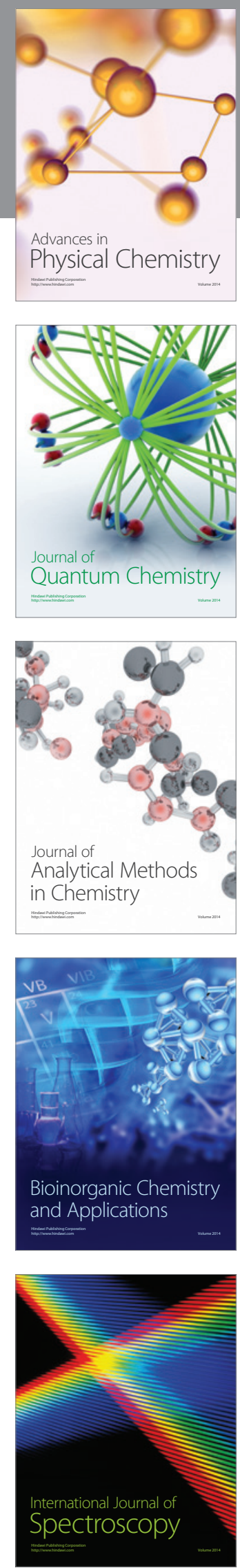